\title{
CONVERGENT NETS OF PARABOLIC AND GENERALIZED SUPERPARABOLIC FUNCTIONS
}

\author{
NEIL A. EKLUND
}

ABSTRACT. The well-known convergence properties of families of harmonic functions are generalized to functions which satisfy $L u=0$ where $L$ is the weak parabolic operator in divergence form. Properties of superharmonic functions are obtained for generalized superparabolic functions. These results are obtained on any bounded domain in $E^{n+1}$.

Consider the parabolic operator in divergence form given by

$$
L u=u_{t}-\left\{a_{i j}(x, t) u,_{i}+d_{j}(x, t) u\right\}_{j}-b_{j}(x, t) u,_{j}-c(x, t) u .
$$

In two preceding papers by the author [4], [5], existence, representation, and a maximum principle were obtained for solutions of $L u=0$ in the cylindrical domain $Q=\Omega \times(0, T)$ for $\Omega \subset E^{n}$, and generalized superparabolic functions in $Q$ were introduced. In this article the author will consider convergent nets of parabolic and superparabolic functions on a bounded domain $U$ assumed to be in $E^{n} \times(0, T)$. Since some of the properties obtained in this article will depend on results for superparabolic functions, it is necessary to restate these results for the domain $U$. The numbering of definitions and theorems in this article continues from those in [5].

Definition 7. Let $z=(x, t), w=(y, s) \in U . z<w$ in $U$ if there is a polygonal path $\left\{C_{z, w}(\alpha), 0 \leq \alpha \leq 1\right\}$ such that

(1) $C_{z, w}(0)=\{z\}, C_{z, w}(1)=\{w\}$,

(2) if $C_{z, w}(\alpha)=\left\{\left(\xi_{\alpha}, \tau_{\alpha}\right)\right\}$, then $\alpha<\beta$ implies $\tau_{\alpha} \leq \tau_{\beta}$,

(3) $\left\{C_{z, w}(\alpha) ; 0 \leq \alpha \leq 1\right\} \subset U$.

Note that Definitions 4,5 , and 6 can be generalized to the domain $U$ since all properties are in terms of standard rectangles in the given domain. Accordingly, let $\mathcal{L}_{U}, \mathcal{S}_{U}^{\prime}, \mathcal{S}_{U}^{\prime \prime}$, and $\mathcal{S}_{U}^{\prime \prime}$ denote the corresponding spaces. The

Received by the editors April 3, 1974.

AMS (MOS) subject classifications (1970). Primary 35K10; Secondary 31C99.

Key words and phrases. Parabolic operator, sequences, generalized superparabolic functions, parabolic minorants. 
theorems and corollaries stated below correspond to the analogous results obtained for $Q$ in [5].

Theorem 4'. If $u \in \mathcal{S}_{U}^{\prime \prime \prime}$ and if there is a $z_{0} \in U$ sucb that $0 \geq u\left(z_{0}\right)=$ $\inf _{U} u$, then $u(z)=u\left(z_{0}\right)$ for all $z \prec z_{0}$ in $U$.

The following corollary is the analogue of that preceding Theorem 7 in [5].

Corollary. If $u \in \mathcal{S}_{U}^{\prime}$, then

(1) $u\left(z_{0}\right)<\infty$ implies $u(z)<\infty$ for all $z \prec z_{0}$ in $U$;

(2) $u\left(z_{0}\right)=\infty$ implies $u(z)=\infty$ for all $z_{0}<z$ in $U$.

Theorem 10'. Let $u \in \mathcal{S}_{U}^{\prime}$ and let $R$ be a standard rectangle in $U$. If $u<\infty$ on $R$, then $L(u ; z, R)$ exists and the function

$$
v(z)= \begin{cases}u(z), & z \in U-R, \\ L(u ; z, R), & z \in R,\end{cases}
$$

satisfies $u \geq v, L v=0$ on $R$, and $v \in \mathcal{S}_{U}^{\prime}$.

Lemma 2. If $\left\{u_{a} ; a \in A\right\}$ is a net of functions parabolic on $U$ wbich converges uniformly on $U$ to $u$, then $u$ is parabolic on $U$.

Proof. For any standard rectangle $R, \bar{R} \subset U$, and any $z \in R, u_{a}(z)=$ $L\left(u_{\alpha} ; z, R\right)$. Since $u_{\alpha} \rightarrow u$ uniformly on $R$, it follows that $u(z)=L(u ; z, R)$. Since $R$ in $U$ was arbitrary, $u$ is parabolic on $U$.

Theorem 11. If $\mathcal{F}$ is a family of parabolic functions on $U$ uniformly bounded on a neighborbood of each point of $U$ and if $K$ is compact in $U$, then the family $\mathcal{F}$ is uniformly equicontinuous on $K$ and eacb net $\left\{u_{a} ; a \epsilon\right.$ $A\}$ in $\mathcal{F}$ bas a subnet which converges uniformly on $K$. If $\left\{u_{\alpha} ; \alpha \in A\right\}$ is a convergent net of uniformly bounded parabolic functions on $U$, then $u \equiv$ $\lim _{A} u_{a}$ is parabolic on $U$.

Proof. For each $z \in K$, let $N_{z}$ denote the neighborhood of $z$ on which the family $\mathcal{F}$ is uniformly bounded. Then $\bigcup\left\{N_{z} ; z \in K\right\}$ is an open cover for $K$ and, by the Heine-Borel theorem, there is a finite subcover $N$. Let $N=$ $\bigcup_{j=1}^{k}\left\{N_{z} ; z=z_{j} \in K\right\}$. Then the family $\mathcal{F}$ is uniformly bounded on $N$ (and hence on $K)$. Each member of $\mathcal{F}$ is parabolic on $U$ and, hence, is Hölder continuous on $U$. Since $K$ is compact in $U$, the Hölder coefficients and exponents can be made uniform for the family $\mathcal{F}$ and it follows that $\mathcal{F}$ is an equicontinuous family on $K$. It follows from Arzela's theorem that each net $\left\{u_{\alpha} ; \alpha \in A\right\}$ in $\mathcal{F}$ has a uniformly convergent subnet on $K$.

Let $\left\{u_{\alpha} ; \alpha \in K\right\}$ be a convergent net of uniformly bounded parabolic 
functions on $U$. Then $u \equiv \lim _{A} u_{\alpha}$ exists. Let $\left\{K_{j}\right\}$ be a sequence of compact sets in $U$ such that $K_{j} \uparrow U$. It follows from what has been shown that for $K_{1}$ there is a subnet $\left\{u_{a}\right\}_{1}$ of $\left\{u_{a} ; \alpha \in A\right\}$ which converges to a parabolic function on $K_{1}$. Since $u_{a} \rightarrow u$ on $U, u$ is parabolic on $K_{1}$. Using what has been shown on $K_{2}$, there is a subnet $\left\{u_{\alpha}\right\}_{2}$ of $\left\{u_{\alpha}\right\}_{1}$ which converges to a parabolic function, $u$, on $K_{2}$. Continuing this process, the diagonalization process can be used to see that $u$ is parabolic on all compact subsets of $U$ and, hence, on $U$ itself.

Definition 8. Let $u$ be defined on $U$. Let $U_{P}=\{z \in U ; u$ locally parabolic at $z\}$ and $U_{ \pm}=\{z \in U ; u(z)= \pm \infty\}$. $u$ is said to have property $P_{ \pm}$if

(1) $w \in U_{P}$ implies $z \in U_{P}$ for all $z \in U$ with $z \prec w$.

(2) $w \in U_{ \pm}$implies $z \in U_{ \pm}$for all $z \in U$ with $z>w$.

(3) $U-U_{P}-U_{+}-U_{-}$is a finite union of sets of the form $\Omega_{j} \times\left(t_{j}\right)$.

Theorem 12. If $u_{j}$ is an increasing (or decreasing ) sequence of parabolic functions on $U$, then $u(z)=\lim u_{j}(z)$ satisfies property $P_{+}\left(\right.$or $\left.P_{-}\right)$.

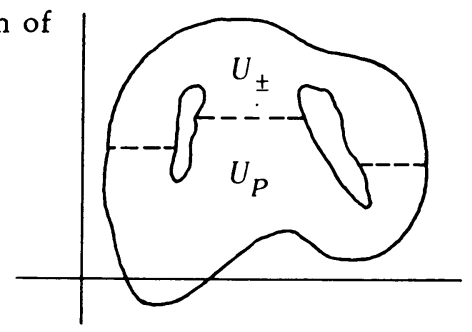

Proof. Assume $u_{j} \uparrow u$. If for some $w \in U, u(w)<\infty$, then for any standard rectangle $R$ with $\bar{R} \subset U$ and $w \in R$, the monotone convergence theorem implies that

$$
L(u ; w, R)=\lim L\left(u_{j} ; w, R\right)=\lim u_{j}(w)=u(w)<\infty
$$

and, hence, $u(z)<\infty$ on $\partial_{p} R$ with $z<w$. Therefore, $U_{P}$ satisfies property (1) of Definition 8. Since $u \geq u_{1} \geq-\infty$ by the parabolicity of $u_{1}, U_{-}=\varnothing$. To see that $U_{+}$satisfies property (2), suppose $u(w)=+\infty$ and suppose for some $z \in U$ with $w<z$ that $u(z)<\infty$. Then by our first argument $u(w)<\infty$ and a contradiction is obtained. Therefore property (2) is satisfied. Since $u_{j} \uparrow u$, for any $z \in U$ either $u(z)<\infty$ or $u(z)=\infty$ must hold. Hence, property (3) is vacously satisfied.

Definition 9. A family $\mathcal{F}$ of functions defined on $U$ is right-directed if for each pair $u, v \in \mathcal{F}$ there is a function $w \in \mathcal{F}$ such that $u \leq w$ and $v \leq w$. $\mathcal{F}$ is left-directed if the above inequalities are reversed.

Lemma 3. If $\left\{u_{i} ; i \in I\right\}$ is a right-directed (left-directed) family of functions parabolic on $U_{\text {, then }} u \equiv \sup _{I} u_{i}\left(u \equiv \inf _{I} u_{i}\right)$ satisfies property $P_{+}\left(P_{-}\right)$ in $U$.

Proof. If $\left\{u_{i} ; i \in I\right\}$ contains only one function then we are done. If 
$\left\{u_{i} ; i \in I\right\}$ contains two or more functions, then the right-directedness can be used to obtain a sequence $\left\{u_{j}\right\}$ in $\left\{u_{i} ; i \in I\right\}$ such that $u_{j} \uparrow u \equiv \sup _{I} u_{i}$. The desired result follows from Theorem 12.

Definition 10. A family $\mathcal{F}$ in $\mathcal{S}_{U}^{\prime}$ is saturated if

(1) $u, v \in \mathcal{F} \Rightarrow u \wedge v \in \mathcal{F}$,

(2) $u \in \mathcal{F} \Rightarrow u^{*} \in \mathcal{F}$ where, for some standard rectangle $R$ in $U$,

$$
u^{*}(z)= \begin{cases}u(z) & \text { in } U-R, \\ L(u ; z, R) & \text { in } R .\end{cases}
$$

Theorem 13. If $\mathcal{F}$ is a saturated family in $\mathcal{S}_{U}^{\prime}$, then $v \equiv \inf _{\mathcal{F}} u$ satis.

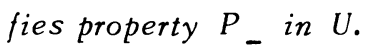

Proof. Let $R$ be an arbitrary standard rectangle. It will be shown that $v$ satisfies property $P_{-}$on $R$ and the desired result will follow from the arbitrariness of $R$ in $U$.

For each $u \in \mathcal{F}$, let $u^{*}$ be defined as in (2). By Theorem $10^{\prime}, u^{*} \leq u$ on $R$. Moreover, since $\mathcal{F}$ is saturated in $\mathcal{S}_{U^{\prime}}^{\prime}, u \in \mathcal{F}$ implies $u^{*} \in \mathcal{F}$. Therefore,

$$
v=\inf \{u ; u \in \mathcal{F}\}=\inf \left\{u^{*} ; u \in \mathcal{F}\right\}
$$

Let $\mathcal{F}^{*}=\left\{u^{*} ; u \in \mathcal{F}\right\}$. If it can be shown that $\mathfrak{F}^{*}$ is a left-directed family, the desired result will follow from Lemma 3. Let $u, w \in \mathcal{F}$. Then $u \wedge w \in \mathcal{F}$ and hence $u^{*}, w^{*}$, and $(u \wedge w)^{*} \in \mathcal{F}$. However $u \wedge w \leq u$ implies $(u \wedge w)^{*} \leq$ $u^{*}$ and similarly $(u \wedge w)^{*} \leq w^{*}$. That is, for any two elements $u^{*}, w^{*} \in \mathcal{F}^{*}$, there is an element, namely $(u \wedge w)^{*}$, in $\mathcal{F}$ such that $(u \wedge w)^{*} \leq u^{*}$ and $(u \wedge w)^{*} \leq w^{*}$. Hence, $\mathfrak{F}^{*}$ is left-directed and the proof is complete.

The preceding results for families of parabolic and generalized superparabolic functions will next be used to find the greatest parabolic minorant of a given function $u$ on $U$. Let $W$ be an open set in $U$ and let $u \in \mathcal{S}_{U}^{\prime}$. Let $\left\{R_{j}\right\}$ be a sequence of standard rectangles satisfying

(1) $\bar{R}_{j} \subset W$ for all $j$,

(2) $W=\bigcup_{j=1} R_{j}$,

(3) each rectangle $R_{j}$ occurs infinitely often in the sequence $\left\{R_{j}\right\}$.

Define

$$
u_{1}(z)= \begin{cases}u(z), & z \in U-R_{1}, \\ L\left(u ; z, R_{1}\right), & z \in R_{1},\end{cases}
$$

and inductively 


$$
u_{n}(z)= \begin{cases}u_{n-1}(z), & z \in U-R_{n}, \\ L\left(u_{n-1} ; z, R_{n}\right), & z \in R_{n^{\prime}}\end{cases}
$$

Then by Theorem $10^{\prime} u_{n} \leq u_{n-1}$ on $U, u_{n}$ is parabolic on $R_{n}$, and $u_{n} \in \mathcal{S}_{U^{*}}^{*}$. Since $\left\{u_{n}\right\}$ is a decreasing sequence, $u_{\infty}(z) \equiv \lim _{n \rightarrow \infty} u_{n}(z)$ satisfies property $P_{-}$on $U$.

Definition 11. $u_{\infty}$ is called the reduction of $u$ over $W$ relative to $U$.

It appears as if for a given open set $W$ in $U$ the corresponding reduction $u_{\infty}$ will depend on the defining sequence $\left\{R_{j}\right\}$. It will follow from Theorem 15 that $u_{\infty}$ is independent of the choice of $\left\{R_{j}\right\}$ if there is a parabolic function $v$ on $W$ with $v \leq u$.

Theorem 14. If $u \in \mathcal{S}_{U}^{\prime}, W$ is open in $U$, and $u_{\infty}$ is the reduction of $u$ over $W$ relative to $U$ corresponding to the sequence $\left\{R_{j}\right\}$, then $u_{\infty}$ satisfies property $P_{-}$on eacb component of $W$, and $u_{\infty}=u$ on $U-W$.

Proof. Without loss of generality assume $W$ is connected. For each $j$,

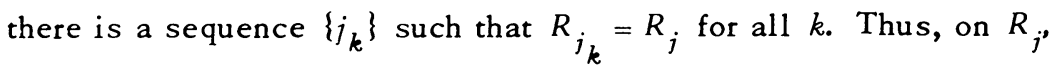

$$
u_{\infty}(z)=\lim _{k \rightarrow \infty} u_{j_{k}}(z)
$$

Since $u_{j}$ is parabolic on $R_{j}$ for all $k$, it follows from Theorem 12 that $u_{\infty}$ satisfies property $P_{-}$on $R_{j}$. Using this procedure on each different rectangle $R_{j}$ in the defining sequence, it follows that $u_{\infty}$ satisfies property $P_{-}$on $W$. Since $u_{j} \equiv u$ on $U-W$ for all $j, u_{\infty}=u$ on $U-W$.

Lemma 4. If $u \in \mathcal{S}_{U}^{\prime}, W$ is open in $U, v$ is parabolic on $W$, and $v \leq u$ on $W$, then $v \leq u_{\infty} \leq u$ on $W$ where $u_{\infty}$ is the reduction of $u$ over $W$ in $U$.

Proof. Let $\left\{R_{j}\right\}$ be the sequence of standard rectangles which define the reduction of $u$ over $W, u_{\infty}$. Then

$$
v(z)=L\left(v ; z, R_{1}\right) \leq L\left(u ; z, R_{1}\right)=u_{1}(z) \leq u(z) \text { on } R_{1},
$$

and

$$
v(z) \leq u(z)=u_{1}(z) \text { on } W-R_{1} .
$$

Therefore, $v \leq u_{1} \leq u$ on $W$. Proceeding inductively, the desired result is obtained.

Definition 12. If $u \in \mathcal{S}_{U}^{\prime}, v$ is parabolic on $U$, and $v \leq u$ on $U$, then $v$ is called a parabolic minorant of $u . v$ is the greatest parabolic minorant of $u$ if $v$ is a parabolic minorant of $u$ and any other parabolic minorant of $u$ is $\leq v$. 
Theorem 15. If $v \in \mathcal{S}_{U}^{\prime}, W$ is open in $U$, and $u$ bas a parabolic minorant $v$ on $W$, then $u$ bas a unique greatest parabolic minorant on $W$; namely, $u_{\infty}$

Proof. It follows from Lemma 4 that $v \leq u_{\infty} \leq u$ on $W$. But $v$ parabolic on $W$ implies $v>-\infty$ on $W$. Therefore, since $u_{\infty}$ satisfies property $P_{-}$ on $W, u_{\infty}$ is parabolic on $W$. If there were two such $u_{\infty}$, say $u_{\infty, 1}$ and $u_{\infty, 2}$, then $u_{\infty, 1} \leq u_{\infty, 2} \leq u$ on $W$ since $u_{\infty, 1}$ is a parabolic minorant and $u_{\infty, 2} \leq u_{\infty, 1} \leq u$ on $W$ since $u_{\infty, 2}$ is a parabolic minorant. Therefore, $u_{\infty}$ is unique and it is the greatest parabolic minorant of $u$.

Theorem 16. If $u, v \in \mathcal{S}_{U}^{\prime}$ bave barmonic minorants, then $(u+v)_{\infty}=$ $u_{\infty}+v_{\infty}$

Proof. Since $u$ and $v$ have harmonic minorants, $u+v$ does also and, hence, $u_{\infty}, v_{\infty}$, and $(u+v)_{\infty}$ are independent of the defining sequence $\left\{R_{j}\right\}$. For such a sequence and $j \geq 1$

$$
\begin{aligned}
(u+v)_{j}(x) & = \begin{cases}(u+v)_{j-1}(z) & \text { on } U-R_{j}, \\
L\left((u+v)_{j-1} ; z, R_{j}\right) & \text { on } R_{j},\end{cases} \\
& =\left\{\begin{array}{ll}
u_{j-1}(z) & \text { on } U-R_{j} \\
L\left(u_{j-1} ; z, R_{j}\right) & \text { on } R_{j}
\end{array}\right\}+\left\{\begin{array}{ll}
v_{j-1}(z) & \text { on } U-R_{j} \\
L\left(v_{j-1} ; z, R_{j}\right) & \text { on } R_{j}
\end{array}\right\} \\
& =u_{j}(z)+v_{j}(z) .
\end{aligned}
$$

Since $u_{j} \downarrow u_{\infty}$ and $v_{j} \downarrow v_{\infty}$, it follows that $(u+v)_{j} \downarrow(u+v)_{\infty}$ and $(u+v)_{\infty}$ $=u_{\infty}+v_{\infty}$

\section{REFERENCES}

1. D. G. Aronson, Non-negative solutions of linear parabolic equations, Ann. Scuola Norm. Sup. Pisa (3) 22 (1968), 607-694.

2. D. G. Aronson and James Serrin, Local behavior of solutions of quasilinear parabolic equations, Arch. Rational Mech. Anal. 25 (1967), 81-122. MR 39 \#5952.

3. Neil Eklund, Boundary behavior of solutions of parabolic equations with discontinuous coefficients, Bull. Amer. Math. Soc. 77 (1971), 788-792. MR 45 \# 2311.

4. - Existence and representation of solutions of parabolic equations, Proc. Amer. Math. Soc. 47 (1975), 137-142.

5. - Generalized super solutions of parabolic equations (submitted).

6. Avner Friedman, Partial differential equations of parabolic type, PrenticeHall, Englewood Cliffs, N. J., 1964. MR 31 \#6062. 
7. O. A. Ladyženskaja and N. N. Ural'ceva, Linear and quasi-linear equations of elliptic type, "Nauka", Moscow, 1964; English transl., Academic Press, New York, 1968. MR 35 \# 1955; 39 \#5941.

8. Walter Rudin, Real and complex analysis, McGraw-Hill, New York, 1966. MR $35 \# 1420$.

9. Neil Trudinger, Pointwise estimates and quasilinear parabolic equations, Comm. Pure Appl. Math. 21 (1968), 205-226. MR 37 \# 1758.

DEP ARTMENT OF MATHEMATICS, VANDERBILT UNIVERSITY, NASHVILLE, TENNESSEE 37235

Current address: Department of Mathematics, Centre College, Danville, Kentucky 40422 